\title{
Zero curvature conditions and conformal covariance
}

\author{
G. Akemann \\ Institut für Theoretische Physik, Universität Hannover, Appelstrasse 2, D-3000 \\ Hannover 1, Germany \\ R. Grimm \\ Laboratoire de Physique Théorique ENSLAPP, ${ }^{\text {a) }}$ Chemin de Bellevuc BP 110, F-74941 \\ Annecy-le-Vieux Cedex, France
}

(Received 25 June 1992; accepted for publication 24 August 1992)

Two-dimensional zero curvature conditions with special emphasis on conformal properties are investigated in detail and the appearance of covariant higher order differential operators constructed in terms of a projective connection is elucidated. The analysis is based on the Kostant decomposition of simple Lie algebras in terms of representations with respect to their "principal" SL(2) subalgebra.

\section{INTRODUCTION}

The construction of covariant differential operators in terms of projective connections (i.e., quadratic differentials with a Schwarzian derivative as an inhomogeneous term in their transformation law) has attracted a certain amount of interest for some time in the investigation of the integrability properties of nonlinear dynamical systems. The fact that Möbius invariant differential operators expressed in terms of the Schwarzian derivative was employed in Ref. 1 in a general recursive construction (see also Refs. 2 and 3 for a review).

More recently, aiming at a clarification of the relation between integrable systems and conformal field theory, the issue of the construction of such covariant differential operators in relation with $W$ algebras has been addressed in Refs. $4-6$ based essentially on the arguments of Drinfeld and Sokolov. ${ }^{7}$

Among other things it was the appearance of structures of integrable hierarchies in the matrix model formulation of two-dimensional gravity which has led to a strong belief that deep connections between integrable models and two-dimensional conformal field theories should exist, calling for reconciliation of integrable hierarchies with conformal structures.

Already some time ago Gervais and Neveu pointed out a close analogy between the Virasoro algebra and the second Hamiltonian structure occurring in the description of the Korteweg-de Vries (KdV) hierarchy equation. ${ }^{8-10}$ This observation was generalized subsequently to the supersymmetric case ${ }^{11}$ and further pursued in Ref. 12.

More recently, relations between the SL(2) zero curvature formulation of integrable systems and the anomalous Ward identities for the energy-momentum tensor have been proposed in Refs. 13 and 14, along with a generalization to the corresponding $\operatorname{SL}(n)$ construction and its possible interpretation in terms of conformal Ward identities of $W_{n}$ gravity.

In this paper we study, in a rather elementary way, two-dimensional zero curvature conditions with special emphasis on the issue of conformal covariance. As is well-known, the basic object in the context of two-dimensional conformal structures is the Beltrami differential which serves to parametrize inequivalent conformal structures in a globally well-defined way. As such, it is clear that the Beltrami differential plays an important role in string theory, respectively, conformal field theory where it couples to the energy-momentum tensor.

For these reasons it will be the central object in our analysis as well. Our demarche is

\footnotetext{
'URA 14-36 du CNRS, associée à l'E.N.S. de Lyon, et au L.A.P.P.
} 
straightforward and to a large extend self-contained. In a first step, in Sec. II, we review in some detail the case of SL(2) from our point of view. We assign conformal properties to the gauge potentials such that holomorphic coordinate transformations are included in a concise way. The notion of Schwarzian derivative and projective connection is shown to arise in a very natural way in this setting.

In Sec. III we investigate the general case, using the Kostant decomposition of simple Lie algebras: ${ }^{15}$ the generators are arranged in representations with respect to the "principal" SL(2) subalgebra. The conformal covariance of the complete zero curvature structure written in this basis is then established by means of the assignment of conformal properties in the SL(2) subsector, as defined in Sec. II. In each representation, labeled by SL(2) spin s, occuring in this decomposition, the gauge potentials are constraint such that just two conformally covariant entities remain: one at lowest weight, which generalizes the Beltrami differential and one at highest weight, a $W$ primary field of spin $s+1$. We then point out how the higher order covariant differential operators arise through an explicit recursive procedure.

In Sec. IV, as an illustration of the general discussion we present the example of SL (3) in some detail, making contact with earlier investigations ${ }^{13,14}$ and extent our methods to discuss then the infinite dimensional wedge subalgebra of $w_{1+\infty}$ along the same lines.

In Sec. $V$ we proceed with $O S p(1 \mid 2)$ as a supersymmetric example, which is in a rather obvious way the generalization of the SL(2) construction, given the Beltrami parametrization of the corresponding superspace geometry. ${ }^{16,17}$

\section{SL(2) GAUGE STRUCTURE AND CONFORMAL COVARIANCE}

Consider the differential one-forms

$$
\Omega=m^{k} L_{k}
$$

in two dimensions which take their values in the Lie algebra of SL(2), i.e.,

$$
\left[L_{k}, L_{l}\right]=(k-l) L_{k+l},
$$

for the values $k, l=-1,0,+1$. The $m^{k}$ are differential onc-forms in two dimensions, parametrized as follows:

$$
m^{k}=d z m_{z}^{k}+d \bar{z} m_{\bar{z}}^{k}
$$

Gauge transformations are defined as

$$
8 \Omega=g \Omega g^{-1}+g d g^{-1},
$$

where $g=g\left(\alpha^{-}, \alpha^{0}, \alpha^{+}\right)$. With these definitions the covariant field strength takes the form

$$
F=d \Omega-\Omega \Omega=F^{k} L_{k},
$$

with coefficients

$$
F^{m}=d m^{m}+l m^{k} m^{l} \delta_{l+k}^{m},
$$

and summation over repeated indices is understood. In some more detail we have

$$
\begin{aligned}
& F^{-1}=d m^{-1}+m^{-1} m^{0}, \\
& F^{0}=d m^{0}+2 m^{-1} m^{+1},
\end{aligned}
$$




$$
F^{+1}=d m^{+1}-m^{+1} m^{0}
$$

We impose the zero curvature condition

$$
F^{k}=0
$$

and identify

$$
m^{-1}=\mu^{z}=d z+d \bar{z} \mu_{\bar{z}}^{z}
$$

This choice assigns conformal weight -1 to $m^{-1}$ and triggers the conformal properties of $m^{0}$ and $m^{+1}$ as well. First of all, requiring the conformal weights of the various terms in the zero curvature condition to match correctly leads us to parametrize

$$
m^{0}=\chi=d z \chi_{z}+d \bar{z} \chi_{\bar{z}}
$$

and

$$
m^{+1}=\lambda_{z}=d z \lambda_{z z}+d \bar{z} \lambda_{\bar{z} z}
$$

In this notation the zero curvature conditions take the form

$$
\begin{aligned}
& d \mu^{z}+\mu^{z} \chi=0 \\
& d \chi+2 \mu^{z} \lambda_{z}=0 \\
& d \lambda_{z}-\lambda_{z} \chi=0
\end{aligned}
$$

Due to the constant term in the definition of $\mu^{z}$, the quadratic terms in the first two equations actually contain linear pieces. As a consequence, the coefficients $\chi_{\bar{z}}$ and $\lambda_{\bar{z} z}$ can be expressed as

$$
\begin{gathered}
\chi_{\bar{z}}=\left(\partial_{z}+\chi_{z}\right) \mu_{\bar{z}}^{z}, \\
\lambda_{\bar{z} z=}=\mu_{\bar{z}}^{z} \lambda_{z z}+\frac{1}{2} \partial_{z}\left(\partial_{z}+\chi_{z}\right) \mu_{\bar{z}}^{z}-\frac{1}{2} \partial_{\bar{z}} \chi_{z}
\end{gathered}
$$

Finally, substituting for $\chi_{\bar{z}}$ and $\lambda_{\bar{z} z}$ in the third equation yields, with very little algebraic effort

$$
\partial_{\bar{z}}\left(\lambda_{z z}+\frac{1}{2} \partial_{z} \chi_{z}-\frac{1}{4} \chi_{z} \chi_{z}\right)=2 \lambda_{z z} \partial_{z} \mu_{\bar{z}}^{z}+\mu_{\bar{z}}^{z} \partial_{z} \lambda_{z z}+\frac{1}{2}\left(\partial_{z}-\chi_{z}\right) \partial_{z}\left(\partial_{z}+\chi_{z}\right) \mu_{\bar{z}}^{z}
$$

In the light of the analysis presented so far, it is suggestive to establish conformal covariance in requiring that $\chi_{z}$ transforms as a gauge potential under holomorphic changes of coordinates $z \rightarrow w(z)$ such that $\partial_{z}+\chi_{z}$ acts as a covariant derivative on $\mu_{\bar{z}}^{z}$ [recall here the special SL(2) gauge transformation of parameter $\alpha^{0}$, i.e., ${ }^{g\left(\alpha^{0}\right)} m^{-1}=m^{-1} \exp \left(+\alpha^{0}\right)$, $\left.{ }^{g\left(\alpha^{0}\right)} m^{0}=m^{0}-d \alpha^{0},{ }^{g\left(\alpha^{0}\right)} m^{+1}=m^{+1} \exp \left(-\alpha^{0}\right)\right]$. In more explicit terms, we assign transformation laws

$$
\mu_{\bar{w}}^{w}=\frac{w^{\prime}}{\bar{w}^{\prime}} \mu_{\bar{z}}^{z}
$$

and 


$$
\chi_{w}=\frac{1}{w^{\prime}}\left(\chi_{z}-\frac{w^{\prime \prime}}{w^{\prime}}\right)
$$

with primes attached to $w$ denoting derivatives with respect to $z$. Moreover, $\lambda_{z z}$ transforms as a quadratic differential, i.e.,

$$
\lambda_{w w}=\left(\frac{1}{w^{\prime}}\right)^{2} \lambda_{z z} .
$$

As is well-known, ${ }^{17}$ given this transformation law for $\chi_{z}$, the combination

$$
\pi_{z z}=\partial_{z} \chi_{z}-\frac{1}{2} \chi_{z} \chi_{z}
$$

transforms as a projective connection, i.e.,

$$
\pi_{w w}=\frac{1}{\left(w^{\prime}\right)^{2}}\left(\pi_{z z}-S_{z z}(w)\right)
$$

with the Schwarzian derivative,

$$
S_{z z}(w)=\left(\frac{w^{\prime \prime}}{w^{\prime}}\right)^{\prime}-\frac{1}{2}\left(\frac{w^{\prime \prime}}{w^{\prime}}\right)^{2},
$$

as inhomogeneous term.

At this stage, a simple reshuffling of terms in the third order covariant derivative appearing above allows to establish the identity (as a simple example of more general structures ${ }^{1,3,5}$ )

$$
\left(\partial_{z}-\chi_{z}\right) \partial_{z}\left(\partial_{z}+\chi_{z}\right) \mu_{\bar{z}}^{z}=\left(\partial_{z} \partial_{z} \partial_{z}+\partial_{z} \cdot \pi_{z z}+\pi_{z z} \partial_{z}\right) \mu_{\bar{z}}^{z}
$$

This shows in particular that in the final zero curvature equation only the combination

$$
\Lambda_{z z}=2 \lambda_{z z}+\pi_{z z}
$$

of the "projective connection" $\pi_{z z}$ and the quadratic differential $\lambda_{z z}$ appears such that we are simply left with

$$
\partial_{\bar{z}} \Lambda_{z z}=\left(\partial_{z} \partial_{z} \partial_{z}+\partial_{z} \cdot \Lambda_{z z}+\Lambda_{z z} \partial_{z}\right) \mu_{\bar{z}}^{z}
$$

We have thus shown that the third order differential operator

$$
\Delta^{(3)}=\partial_{z} \partial_{z} \partial_{z}+\partial_{z} \cdot \Lambda_{z z}+\Lambda_{z z} \partial_{z}
$$

acts covariantly on tensors of conformal weight minus one, provided $\Lambda_{z z}$ transforms inhomogeneously with a Schwarzian derivative. The disappearance of $\chi_{z}$ corresponds to a special SL(2) gauge transformation of parameter $2 \alpha^{+}=-\chi_{z}$, which, when acting on $\lambda_{z z}$ has the form of a Miura transformation. ${ }^{3}$

Observe that the same differential operator appears also in the second Hamiltonian structure of the $\mathrm{KdV}$ equation.

As to possible relations between the anomalous conservation equation of the energy momentum tensor in two-dimensional conformal theory and the $\mathrm{KdV}$ hierarchy we refer to Refs. 13 and 14. 
Here we wish to point out shortly, that the structures presented so far can be easily generalized such that a consistent Becchi-Rouet-Stora (BRS) differential algebra can be derived geometrically, in imitation of the gauge and ghost field geometry of Yang-Mills theory.

The prescription consists in enlarging the space of differential forms considered so far, in taking into account geometrically ghost fields, i.e.,

$$
\tilde{\mu}^{z}=\mu^{z}+c^{z}, \quad \tilde{\chi}=\chi+c, \quad \tilde{\lambda}_{z}=\lambda_{z}+c_{z},
$$

and defining the generalized exterior derivative $\tilde{d}=d+s$. The zero curvature conditions in this enlarged setting then read

$$
\begin{aligned}
& \tilde{d} \tilde{\mu}^{z}+\tilde{\mu}^{z} \tilde{\chi}=0, \\
& \tilde{d} \tilde{\chi}+2 \tilde{\mu}^{z} \tilde{\lambda}_{z}=0, \\
& \tilde{d} \tilde{\lambda}_{z}-\tilde{\lambda}_{z} \tilde{\chi}=0 .
\end{aligned}
$$

Going through this set of equations at ghost numbers one and two allows, first of all to express the dependent variables and to show that the ensuing BRS differential algebra closes on $\mu_{\bar{z}}^{z}, c^{z}$, and $\Lambda_{z z}$ in the following simple way (see also Ref. 18):

$$
\begin{gathered}
s \mu_{\bar{z}}^{z}=\partial_{\bar{z}} c^{2}-\mu_{\bar{z}}^{z} \partial_{z} c^{z}+c^{z} \partial_{z} \mu_{\bar{z}}^{z}, \\
s c^{z}=-c^{z} \partial_{z} c^{z}, \\
s \Lambda_{z z}=\left(\partial_{z} \partial_{z} \partial_{z}+\partial_{z} \cdot \Lambda_{z z}+\Lambda_{z z} \partial_{z}\right) c^{z} .
\end{gathered}
$$

This completes the parenthesis on the geometrical BRS structure pertaining to the SL(2) zero curvature condition.

In closing we stress that the discussion of this section is based on the identification of the SL(2) gauge potential $m^{-1}$ with the Beltrami differential $\mu^{z}=d z+d \bar{z} \mu_{\bar{z}}^{z}$, soldering SL(2) gauge transformations with holomorphic coordinate transformations. Then, as a consequence of the covariance of the zero curvature conditions, $\chi_{z}$ is absorbed in a redefinition of $\lambda_{z z}$, leaving as independent fields $\mu_{\bar{z}}^{\bar{z}}$, covariant of conformal weight minus one and $\Lambda_{z z}$, which transforms as a projective connection.

\section{SOME GENERAL CONSIDERATIONS}

The following discussion is based on the fact, ${ }^{15}$ that the generators of any simple Lie algebra $g$ can be arranged in representations with respect to the principal SL(2) subalgebra. In this basis it is convenient to note the generators with a double index according to the SL(2) spin and its third component. At spin $a+1$ we have $2 a+3$ generators $W_{k}^{a}$ with range of indices restricted to

$$
a \geqslant 0, \text { and } \quad-a-1 \leqslant k \leqslant a+1 .
$$

The commutation relations are then defined as

$$
\left[W_{k}^{a}, W_{l}^{b}\right]=c_{k l c}^{a b m} W_{m}^{c}
$$

and we identify the basic SL(2) at $a=0$, i.e., $W_{k}^{0}=L_{k}$ with commutation relations as defined in the preceding section. As to the remaining commutators we shall use the convention $\underline{b} \geqslant 1$ for underlined indices. We parametrize 


$$
\begin{gathered}
{\left[W_{-1}^{0}, W_{l}^{b}\right]=-\sigma_{-}(\underline{b}, l) W_{l-1}^{b},} \\
{\left[W_{0}^{0}, W_{l}^{b}\right]=-l W_{l}^{b},} \\
{\left[W_{+1}^{0}, W_{l}^{b}\right]=-\sigma_{+}(\underline{b}, l) W_{l+1}^{b},}
\end{gathered}
$$

with appropriate structure constants $\sigma_{-}(\underline{b}, l)$ and $\sigma_{+}(\underline{b}, l)$.

The values of SL(2) spins occuring in this decomposition depend on the structure of the particular Lie algebra, they are given in terms of the Lie algebra exponents. ${ }^{15} \Lambda$ glance at the table of exponents (for instance in Ref. 19) shows that a given spin occurs just once, except for the case of $\operatorname{SO}(4 N)$ where spin $2 N-1$ occurs twice. For example, in SL(3) only $a=0,1$, i.e., spins one and two contribute, whereas $G_{2}$ exhibits $a=0,4$, i.e., spins one and five. In the decomposition of $\mathrm{SO}(8)$ spins one and five occur once whereas spin three occurs twice.

We propose here to study zero curvature conditions for Lie algebra valued gauge potentials pertaining to this particular decomposition of the Lie algebra $G$. We define the corresponding one-forms

$$
\Omega=w_{a}^{k} W_{k}^{a}
$$

where summation over the full range of indices is understood, with

$$
w_{a}^{k}=d z w_{z a}^{k}+d \bar{z} w_{\bar{z} a}^{k} .
$$

The corresponding curvatures,

$$
F=d \Omega-\Omega \Omega=F_{a}^{k} W_{k}^{a}
$$

are now explicitly given as

$$
F_{c}^{m}=d w_{c}^{m}-\frac{1}{2} w_{a}^{k} w_{b}^{l} c_{k l c}^{a b}
$$

It is instructive to display separately the field strengths for the values $c=0$ and $c=\underline{c} \geqslant 1$, namely,

$$
F_{0}^{m}=d w_{0}^{m}-k w_{0}^{k} w_{0}^{l} \delta_{l+k}^{m}-\frac{1}{2} w_{\underline{a}}^{k} w_{\underline{b}}^{l} c_{k l}^{a b}{ }_{0}^{m}
$$

and

$$
F_{\underline{c}}^{m}=d w_{\underline{c}}^{m}+m w_{0}^{0} w_{\underline{c}}^{m}+w_{0}^{-1} w_{\underline{c}}^{m+1} \sigma_{-}(\underline{c}, m+1)+w_{0}^{+1} w_{\underline{c}}^{m-1} \sigma_{+}(\underline{c}, m-1)-\frac{1}{2} w_{\underline{a}}^{k} w_{\underline{b}}^{l} c_{k \underline{a}}^{a b} \underline{\underline{c}} .
$$

At $c=0$ we see that additional contributions may appear compared to the pure SL(2) case. It is nevertheless possible to assign consistently conformal weights and maintain conformal covariance very much along the same lines as before. We identify

$$
\begin{gathered}
w_{0}^{-1}=\mu^{z}=d z+d \bar{z} \mu_{\bar{z}}^{z}, \\
w_{0}^{0}=\chi=d z \chi_{z}+d \bar{z} \chi_{\bar{z}}, \\
w_{0}^{+1}=\lambda_{z}=d z \lambda_{z z}+d \bar{z} \lambda_{\bar{z} z} .
\end{gathered}
$$

For $\mu_{\bar{z}}^{z}, \chi_{z}$ and $\lambda_{z z}$ we require the same transformation laws as established in the pure SL(2) case. A consistent scheme is then obtained if the $w_{a}^{k}$ transforms covariantly with weight $k$. 
In the next step we impose the covariant zero curvature conditions

$$
F_{c}^{m}=0
$$

together with the covariant constraints

$$
w_{\underline{a}}^{k}=d \bar{z} w_{\bar{z} \underline{\underline{a}}}^{k}+d z w_{(\underline{\underline{a}}+2)} \delta_{\underline{a}+1}^{k} .
$$

In other words we parametrize $w_{\underline{a}}^{k}$ such that

$$
w_{z \underline{a}}{ }^{k}=0, \text { for } \quad-\underline{a}-1 \leqslant k \leqslant \underline{a},
$$

and we define

$$
w_{2 \underline{\underline{a}}}^{\underline{g}+1}=w_{(\underline{a}+2)} .
$$

The $w_{(\underline{a}+2)}$ are conformally covariant tensors of weight $\underline{a}+2$. For the moment, the constraint equations appear here without any further justification or motivation and should be considered as ansatz, compatible with the conformal structure, which simplify considerably the zero curvature equations, in particular the quadratic terms.

Let us now have a closer look at these zero curvature conditions. From $F_{0}^{m}=0$ we obtain

$$
d w_{0}^{m}-k w_{0}^{k} w_{0}^{l} \delta_{l+k}^{m}-w_{\underline{a}}^{a}+1 w_{\underline{b}}^{l} \underline{c}_{\underline{a}+1}^{a}{ }^{\underline{b}}{ }_{0}^{m}=0 .
$$

Two of these three equations, namely, those at $k=-1$ and $k=0$ serve to determine $\chi_{\bar{z}}$ and $\lambda_{\bar{z} z}$, respectively, while the last one, at $k=+1$, yields a covariant differential equation, which "measures the anholomorphicity of $\Lambda_{z z}$ " It has the form

$$
\partial_{\bar{z}} \Lambda_{z z}=\left(\partial_{z} \partial_{z} \partial_{z}+\partial_{z} \cdot \Lambda_{z z}+\Lambda_{z z} \partial_{z}\right) \mu_{\bar{z}}^{z}+\Xi_{z z z}(\mathrm{~g}),
$$

where the deviation from the pure $\operatorname{SL}(2)$ case is encoded in $\Xi_{\bar{z} z z}(\mathfrak{g})$, which depends, of course, on the details of the Lie algebra considered.

The explicit expressions of the zero curvature conditions $F_{\underline{c}}^{m}=0$ are given as

$$
d w_{\underline{c}}^{m}+m \chi w_{\underline{c}}^{m}+\mu^{z} w_{\underline{c}}^{m+1} \sigma_{-}(\underline{c}, m+1)+\lambda_{z} w_{\underline{c}}^{m-1} \sigma_{+}(\underline{c}, m-1)-w_{\underline{a}}^{a+1} w_{\underline{b}}^{l} \underline{c}_{\underline{a}+1}^{\underline{a}} \stackrel{b}{l}_{\underline{c}}^{m}=0 .
$$

For each $\underline{c}$, there are $2 \underline{c}+3$ equations. We first of all emphasize the appearance of the covariant derivative in terms of $\chi$. Then, for the values up to $m=\underline{c}$ we have $2 \underline{c}+2$ relations, which, due to the presence of the term containing $\mu^{z}$ allow to determine recursively the one-form coefficients $w_{\bar{z} \underline{a}}{ }^{k}$ for $k \geqslant-\underline{c}$. The last equation, at $m=\underline{c}+1$, is then a differential equation which measures the anholomorphicity of the coefficient $w_{(\underline{c}+2)}$, which is a covariant conformal tensor of weight $\underline{c}+2$. In this equation (covariant) derivatives up to order $2 \underline{c}+3$ appear.

Note that, while at $c=0$ we have the fields $\mu_{\bar{z}}^{z}, \chi_{z}$, and $\lambda_{z z}$, at each superior level two new additional independent fields appear, namely, $v_{\bar{z}}^{-(c+1)}=w_{\bar{z} c}-\underline{c-1}$, of conformal weight $-\underline{c}-1$, and $w_{(\underline{c}+2)}$, of conformal weight $\underline{c}+2$. The details of the equations depend of course on the structure of the Lie algebra in question.

So far, covariance was ensured due to the presence of $\chi_{z}$ with transformation law

$$
\chi_{w}=\frac{1}{w^{\prime}}\left(\chi_{z}-\frac{w^{\prime \prime}}{w^{\prime}}\right)
$$


A field dependent special SL(2) gauge transformation $g\left(\alpha^{+}\right)=\exp \left(\alpha^{+} L_{+}\right)$of parameter $2 \alpha^{+}=-\chi_{z}$ eliminates $\chi_{z}$ and replaces $2 \lambda_{z z}$ by

$$
\Lambda_{z z}=2 \lambda_{z z}+\partial_{z} \chi_{z}-\frac{1}{2} \chi_{z} \chi_{z}
$$

which, as we have seen in the preceding section, transforms with Schwarzian derivative as an inhomogeneous term.

On the other hand it leaves invariant the one-form coefficients $w_{\bar{z} \underline{\underline{g}}}^{-\underline{q}-1}$ and $w_{(\underline{g}+2)}$ together with the constraints imposed above, i.e.,

$$
w_{z \underline{a}}{ }^{k}=0, \quad \text { for } \quad-\underline{a}-1 \leqslant k \leqslant \underline{a} .
$$

But this means that the recursive solution of the zero curvature conditions works out in the same way as before. The difference is that now $\chi_{z}$ has disappeared and that the covariance of the higher order differential operators in the final equation is ensured solely in terms of $\Lambda_{z z}$, which transforms as a projective connection. More explicitly, in this gauge the explicit expressions of the zero curvature conditions $F_{c}^{m}=0$ are given as

$$
d w_{\underline{c}}^{m}+\mu^{z} w_{\underline{c}}^{m+1} \sigma_{-}(\underline{c}, m+1)+m d \bar{z} \partial_{\bar{z}} \mu_{\bar{z}}^{z} w_{\underline{c}}^{m}+\frac{1}{2} d z \Lambda_{z z} w_{\underline{c}}^{m-1} \sigma_{+}(\underline{c}, m-1)-w_{\underline{a}}^{a+1} w_{\underline{b}}^{l} c_{\underline{a}}^{\underline{a}}+1 \underline{b}_{\underline{c}}^{\underline{m}}=0 .
$$

To summarize, the whole system is described in terms of two kinds of variables, corresponding to the highest and lowest values of SL(2) spin, respectively, and visualized as white disks in the following diagram:

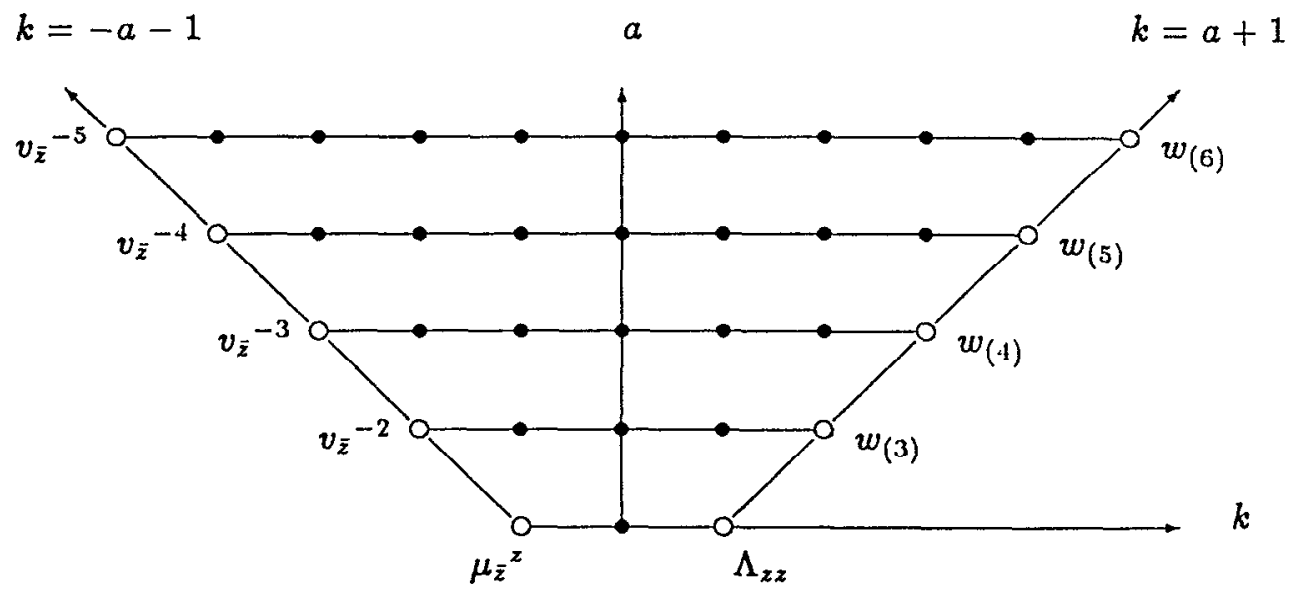

The zero curvature condition above is written as a differential two-form. In order to see its structure in some more detail we explicitly display its coefficient.

It is convenient to consider separately the three cases $m<\underline{c}, m=\underline{c}$, and $m=\underline{c}+1$.

At $m<\underline{c}$ we obtain

$$
w_{\bar{z} \underline{c}}^{m+1} \sigma_{-}(\underline{c}, m+1)=\partial_{z} w_{\bar{z} \underline{c}}^{m}-\frac{1}{2} \Lambda_{z z} w_{\bar{z} \underline{c}}^{m-1} \sigma_{+}(\underline{c}, m-1)+w_{(\underline{a}+2)} w_{\bar{z} \underline{b}}^{l} c_{\underline{g}+1}^{\underline{a}} \underline{b}_{\underline{l}}^{m},
$$

where in the last term we have $l=m-\underline{a}-1$, i.e., $l<m$. For a given value $m+1$ this equation expresses the coefficients $w_{\overline{z c}}^{m+1}$ in terms of coefficients at lower values of $m$, the projective connection $\Lambda_{z z}$, and certain covariant tensors $w_{(\underline{a}+2)}$. In the last term SL(2) spins different from $\underline{c}$ can contribute, the explicit structure depends of course on the properties of the Lie algebra chosen. Hence this string of equations serves to recursively eliminate $w_{\bar{z} c}{ }^{m}$ for $-\underline{c} \leqslant m \leqslant+\underline{c}$ at each value $\underline{c}$ in favor of the covariant tensors $v_{\bar{z}}{ }^{-(\underline{a}+1)}, w_{(\underline{a}+2)}$ and the projective connection $\Lambda_{z z}$. The same kind of remark applies to the equation at $m=\underline{c}$, where we obtain 


$$
\begin{aligned}
& w_{\bar{z} \underline{\underline{c}} \underline{c}+1} \sigma_{-}(\underline{c}, \underline{c}+1)-\mu_{\bar{z}}^{z} w_{(\underline{c}+2)} \sigma_{-}(\underline{c}, \underline{c}+1)=\partial_{z} w_{\bar{z} \underline{c} \underline{c}}-\frac{1}{2} \Lambda_{z z} w_{\bar{z} \underline{c}} \underline{c}-1 \sigma_{+}(\underline{c}, \underline{c}-1) \\
& +w_{(\underline{a}+2)} w_{\bar{z} \underline{b}} c_{\underline{a}+1}^{a} \underline{b} \underline{c} \underline{c}
\end{aligned}
$$

with $l=\underline{c}-\underline{a}-1$. We have written this equation separately because an extra term involving $\mu_{\bar{z}}^{z}$ appears.

The last equation, at $m=\underline{c}+1$, has then a completely different structure, namely,

$$
\partial_{\bar{z}} w_{(\underline{c}+2)}+(\underline{c}+1) \partial_{\bar{z}} \mu_{\bar{z}}^{z} w_{(\underline{c}+2)}=\partial_{z} w_{\bar{z} \underline{c}} \underline{c}+1-\frac{1}{2} \Lambda_{z z} w_{\bar{z} \underline{c}} \underline{c} \sigma_{+}(\underline{c}, \underline{c})+2 w_{(\underline{a}+2)} w_{\bar{z} \underline{\underline{b}}} \underline{c}-\underline{a} c_{\underline{a}}^{\underline{a}}+1 \underline{b} \underline{\underline{b}}-\underline{\underline{a}} \underline{\underline{c}}+1
$$

We emphasize that, after successive substitution, these relations are given in terms of differential expressions involving only the covariant tensors located on the leftmost and on the rightmost diagonal in the previous diagram. We know, according to the previous discussion of covariance properties, that the these differential expressions are covariant due to the appearance and the special properties of $\Lambda_{z z}$.

We are not aiming here at solving the recursive construction in closed form, which of course depends on the structure constants of the Lie algebra in the particular decomposition we have used here. For SL $(N)$ they are given in Ref. 20, whereas for the other Lie algebras we have not found them in the literature so far. ditions

Observe that for $w_{\bar{z} \underline{c}}^{m}=0$ the whole system collapses into the set of holomorphicity con-

$$
\partial_{\bar{z}} w_{(\underline{c}+2)}=0
$$

which should be compared to those occuring in the construction of WZNW Toda theories. ${ }^{21,22}$ In particular one might speculate that nonvanishing $w_{\bar{z} c}^{m}$ could, in some sense, be attributed to gauged versions of the Toda theory or " $W_{\mathrm{g}}$ gravity" 23 in relation with the Lie algebra $g$ along similar lines as in the pure SL(2) case.

\section{TWO EXAMPLES: SL(3) AND THE WEDGE ALGEBRA}

In order to provide an explicit example illustrating the general procedure described in the previous section, we present here the zero curvature construction for SL (3) in some detail, as a prototype for the $\mathrm{SL}(N)$ case, which can be treated along the same lines without any conceptual complications. ${ }^{13,14}$ As a second example, going beyond the finite case, we discuss then the zero curvature structure defined on the infinite dimensional wedge subalgebra of $w_{1+\infty}$, giving rise to an infinite sequence of covariant differential operators.

\section{A. SL(3)}

The Lie algebra SL(3) is given in terms of $W_{k}^{a}$ for $a=0$ and $a=1$. We define

$$
\begin{gathered}
W_{k}^{0}=L_{k}, \quad k=-1,0,+1, \\
W_{m}^{1}=W_{m}, \quad m=-2,-1,0,+1,+2 .
\end{gathered}
$$

The commutation relations are given as

$$
\begin{gathered}
{\left[L_{k}, L_{l}\right]=(k-l) L_{l+k},} \\
{\left[L_{k}, W_{m}\right]=(2 k-m) W_{m+k},}
\end{gathered}
$$

and

$$
\left[W_{ \pm 2}, W_{\mp 1}\right]=\mp 4 L_{ \pm 1}, \quad\left[W_{ \pm 1}, W_{0}\right]= \pm 2 L_{ \pm 1}
$$




$$
\begin{gathered}
{\left[W_{+2}, W_{-2}\right]=-16 L_{0}, \quad\left[W_{+1}, W_{-1}\right]=+2 L_{0},} \\
{\left[W_{ \pm 2}, W_{ \pm 1}\right]=0, \quad\left[W_{ \pm 2}, W_{0}\right]=0 .}
\end{gathered}
$$

Accordingly, the zero curvature conditions are written in terms of gauge potentials $w_{0}^{k}$, $k=-1,0,+1$, and $w_{1}^{m}, m=-2,-1,0,+1,+2$.

The former will be identified with $\mu^{z}, \chi$, and $\lambda_{z}$ in the gauge $\chi_{z}=0$ (see Ref. 24 for nonvanishing $\chi_{z}$ and subsequent explicit elimination in the final result). We define

$$
\begin{gathered}
w_{0}^{-1}=\mu^{z}=d z+d \bar{z} \mu_{\bar{z}}^{z}, \\
w_{0}^{0}=\chi=d \bar{z} \chi_{\bar{z}}, \\
w_{0}^{+1}=\lambda_{z}=\frac{1}{2} d z \Lambda_{\mathrm{zz}}+d \bar{z} \lambda_{\overline{z z}} .
\end{gathered}
$$

For the latter, at spin two, we suppress the index 1 , i.e., $w^{m}=w_{1}^{m}$, and define

$$
w^{m}=d z w_{z}^{m}+d \bar{z} w_{\bar{z}}^{m}
$$

Following the discussion of the general case in the previous section we impose the constraints

$$
w_{z}^{m}=0, \text { for } \quad-2 \leqslant m \leqslant+1 \text {. }
$$

We then define

$$
w^{-2}=d \bar{z} v_{\bar{z}}^{2 z}
$$

and

$$
w^{+2}=d z w_{(3) \operatorname{zzz}}+d \bar{z} w_{\bar{z}}^{+2} .
$$

The basic quantities are thus

$$
\mu_{\bar{z}}^{z}, \quad \Lambda_{z z}, \quad v_{\bar{z}}^{z z}, \text { and } w_{(3) z z z},
$$

in terms of which all the other coefficients will be expressed by virtue of the zero curvature conditions.

The corresponding picture is in this case

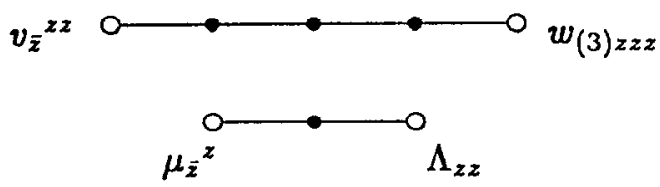

Taking into account explicitly the structure constants displayed above, the three zero curvature conditions at $a=0$ take the form

$$
\begin{gathered}
d \mu^{z}+\mu^{z} \chi=0, \\
d \chi+2 \mu^{z} \lambda_{z}+16 w^{2} w^{-2}=0 \\
d \lambda_{z}-\lambda_{2} \chi+4 w^{2} w^{-1}=0
\end{gathered}
$$

whereas at level $a=1$ we have five equations 


$$
d w^{m}+m \chi w^{m}+(m+3) \mu^{z} w^{m+1}+(m-3) \lambda_{z} w^{m-1}=0,
$$

corresponding to the values $m=-2,-1,0,+1,+2$.

From the first two equations at $a=0$ one determines

$$
\chi_{\bar{z}}=\partial_{\bar{z}} \mu_{\bar{z}}^{z}
$$

and

$$
2 \lambda_{\overline{z z}}=\mu_{\bar{z}}^{z} \Lambda_{z z}+\partial_{z} \partial_{\bar{z}} \mu_{\bar{z}}^{z}-16 v_{\bar{z}}^{z z} w_{(3) z z z}
$$

The third equation is then a differential equation. Before writing it down, however, let us discuss the second set of equations for the $w^{m}$. Note, first of all that, due to the constraints, up to $m=1$ only $\partial_{z}$ derivatives can appear and that the term $m \chi w^{m}$ appears only at $m=2$. For the same reason, in the last term, $(m-3) \lambda_{z} w^{m-1}$, only $\Lambda_{z z}$ can contribute to $\lambda_{z}$, and, most important, the term $(m+3) \mu^{z} w^{m+1}$ is responsible for the recursive determination of the $w_{\bar{z}}^{m}$, i.e.,

$$
\begin{gathered}
w_{\bar{z}}^{-1}=\partial_{\bar{z}} v_{\bar{z}}^{z z}, \\
2 w_{\bar{z}}^{0}=\partial_{\bar{z}} w_{\bar{z}}^{-1}+2 \Lambda_{z z} v_{\bar{z}}^{z z}, \\
3 w_{\bar{z}}^{+1}=\partial_{\bar{z}} w_{\bar{z}}^{0}+\frac{3}{2} \Lambda_{z z} w_{\bar{z}}^{-1}, \\
4 w_{\bar{z}}^{+2}=4 \mu_{\bar{z}}^{z} w_{(3) z z z}+\partial_{z} w_{\bar{z}}^{+1}+\Lambda_{z z} w_{\bar{z}}^{0},
\end{gathered}
$$

the last equation being

$$
\partial_{\bar{z}} w_{(3) z z z}=2 \chi_{\bar{z}} w_{(3) z z z}+\partial_{z} w_{\bar{z}}^{+2}+\frac{1}{2} \Lambda_{z z} w_{\bar{z}}^{+1}
$$

With this information given we arrive, after recursive substitution, at the equations

$$
\partial_{\bar{z}} \Lambda_{z z}=\Delta^{(3)} \mu_{\bar{z}}^{z}-8\left(2 v_{\bar{z}}^{z z} \partial_{z} w_{(3) z z z}+3 w_{(3) z z z} \partial_{\bar{z}} v_{\bar{z}}^{z z}\right)
$$

and

$$
\partial_{\bar{z}} w_{(3) z z z}=\frac{1}{24} \Delta^{(5)} v_{\bar{z}}^{z z}+\mu_{\bar{z}}^{z} \partial_{z} w_{(3) z z z}+3 w_{(3) z z z} \partial_{\bar{z}} \mu_{\bar{z}}^{z}
$$

at $a=0$ and $a=1$, respectively. The third and fifth order covariant differential operators are defined as (we have suppressed here the indices $z$ )

$$
\Delta^{(3)}=\partial^{3}+\partial \Lambda+2 \Lambda \partial
$$

and

$$
\Delta^{(5)}=\partial^{5}+2 \partial^{3} \Lambda+10 \Lambda \partial^{3}+15 \partial \Lambda \partial^{2}+9 \partial^{2} \Lambda \partial+16 \Lambda \partial \Lambda+16 \Lambda \Lambda \partial .
$$

It should be clear that the analysis for the more general case of $\operatorname{SL}(N)$ can be carried out along exactly the same lines without any conceptual complications. The relevant decompositions and commutation relations for $\mathrm{SL}(N)$ are given in Ref. 20. 


\section{B. The wedge algebra}

Next we wish to apply our procedure to the wedge algebra. ${ }^{25}$ It is defined as

$$
\left[W_{k}^{a}, W_{l}^{b}\right]=(k(b+1)-l(a+1)) W_{k+l}^{a+b}
$$

with the range of indices on the generators $W_{k}^{a}$ restricted to

$$
a \geqslant-1 \text {, and }-a-1 \leqslant k \leqslant a+1 \text {. }
$$

In the $k, a$ plane, the (infinitely many) generators are inclosed in the wedge formed from the lines $k=-a-1$ and $k=a+1$. The algebra cannot lead out of the wedge because the commutators of two generators located on the wedge have zero structure constants. Clearly, the commutation relations

$$
\begin{gathered}
{\left[W_{-1}^{0}, W_{l}^{b}\right]=-(b+1+l) W_{l-1}^{b},} \\
{\left[W_{0}^{0}, W_{l}^{b}\right]=-l W_{l}^{b},} \\
{\left[W_{+1}^{0}, W_{l}^{b}\right]=+(b+1-l) W_{l+1}^{b},}
\end{gathered}
$$

show that we are dealing with a decomposition of the infinite dimensional wedge algebra in terms of representations with respect to the SL(2) subalgebra spanned by $W_{-1}^{0}, W_{0}^{0}, W_{+1}^{0}$.

The idea is now again to study zero curvature conditions on the wedge algebra, such that the SL(2) substructure is parametrized in the same way as discussed before, involving the Beltrami differential $\mu_{\bar{z}}^{z}$ and the projective connection $\Lambda_{z z}$.

We begin with the definition of Lie-algebra valued one-forms, i.e.,

$$
\Omega=w_{a}^{k} W_{k}^{a}
$$

where summation over the appropriate range of indices is understood, with

$$
w_{a}^{k}=d z w_{z a}^{k}+d \bar{z} w_{\overline{z a}}{ }^{k}
$$

The corresponding curvatures,

$$
F=d \Omega-\Omega \Omega=F_{a}^{k} W_{k}^{a}
$$

are now explicitly given as

$$
F_{c}^{m}=d w_{c}^{m}+l(a+1) w_{a}^{k} w_{b}^{l} \delta_{k+l}^{m} \delta^{a+b}{ }_{c} .
$$

As anticipated above we assign overall conformal weight $m$ to $w_{c}^{m}$, identify in particular

$$
w_{0}^{-1} \equiv d z+d \bar{z} \mu_{\bar{z}}^{z}
$$

and impose the zero curvature conditions

$$
d w_{c}^{m}+l(a+1) w_{a}^{k} w_{b}^{l} \delta_{k+l}^{m} \delta^{a+b}=0 .
$$

At $c=-1$ this reads simply

$$
d w_{-1}^{0}=0
$$

In the parametrization $w_{-1}^{0} \equiv v=d z v_{z}+d \bar{z} v_{\bar{z}}$ this means 


$$
\partial_{\bar{z}} v_{z}=\partial_{z} v_{\bar{z}}
$$

The zero curvature conditions of the wedge algebra at level $c=0$ reproduce exactly the same equations as in the SL(2) case, which we have already discussed in great detail there, and we use the same notations here. The upshot is then that the Beltrami differential $\mu_{\bar{z}}^{z}$ and the projective connection $\Lambda_{z z}$ are subject to the equation

$$
\partial_{\bar{z}} \Lambda_{z z}=\left(\partial_{\bar{z}} \partial_{z} \partial_{z}+\partial_{z} \cdot \Lambda_{z z}+\Lambda_{z z} \partial_{z}\right) \mu_{\bar{z}}^{z}
$$

For the discussion of the equations at levels $c \geqslant 1$ we follow the strategy of the general case and separate explicitly the indices $c=-1$ and $c=0$. Underlined indices $c$ are then restricted to values $\underline{c}=c \geqslant+1$. In this notation the remaining zero curvature conditions for the wedge algebra take the form

$$
d w_{\underline{c}}^{m}+m \chi w_{\underline{c}}^{m}+l(\underline{a}+1) w_{\underline{a}}^{k} w_{\underline{b}}^{l} \delta_{k+l} \delta^{m} \delta^{\underline{a}+\underline{b}}+(m+\underline{c}+2) \mu^{z} w_{\underline{c}}^{m+1}+(m-\underline{c}-2) \lambda_{z} w_{\underline{c}}^{m-1}=0 .
$$

These equations are still completely general in the sense that no constraints whatsoever have been imposed on the one-forms $w_{\underline{c}}^{m}$. As to the conformal properties, the one-forms $w_{\underline{c}}^{m}$ transform covariantly, with conformal weights $m$. Taking into account the conformal properties already determined for the SL(2) substructure, the first two terms provide just the correct covariant derivative, establishing conformal covariance of the complete zero curvature structure. The SL(2) singlet $w_{-1}^{0}$ has completely decoupled from the system of zero curvature equations.

To proceed we choose the special parametrization where $\chi_{z}=0$ and

$$
w_{z \underline{a}}{ }^{k}=0, \text { for } \quad-\underline{a}-1 \leqslant k \leqslant \underline{a},
$$

at each level $\underline{a}$. The only nonvanishing $z$ component is then denoted

$$
w_{z \underline{a}}^{\underline{a}+1}=w_{(\underline{a}+2)},
$$

indicating its conformal weight $\underline{a}+2$. In this parametrization the zero curvature conditions simplify considerably. To be more explicit, at $m<\underline{c}$ we obtain $(l=m-\underline{a}-1)$

$$
(m+\underline{c}+2){w_{\bar{z} \underline{c}}}^{m+1}=\partial_{\bar{z}}{w_{\bar{z} \underline{c}}}^{m}+\frac{1}{2}(\underline{c}+2-m) \Lambda_{z z} w_{\bar{z} \underline{c}}^{m-1}+(\underline{c}+2-m)(\underline{a}+1) w_{(\underline{a}+2)} w_{\bar{z} \underline{b}}{ }^{l} \delta^{a+b} \underline{c} .
$$

We use then the explicit form of the equation at $m=\underline{c}$ to write that at $m=\underline{c}+1$ as

$$
\begin{aligned}
& \partial_{\bar{z}} w_{(c+2)}-\mu_{\bar{z}}^{z} \partial_{z} w_{(\underline{c}+2)}-(\underline{c}+2) w_{(c+2)} \partial_{z} \mu_{\bar{z}}^{z} \\
& =\frac{1}{2(\underline{c}+1)}\left[\partial_{z} \partial_{z} w_{\bar{z} \underline{\underline{c}} \underline{c}}+(\underline{c}+1) \Lambda_{z z} w_{\bar{z} \underline{\underline{c}} \underline{c}}+\partial_{z}\left(\Lambda_{z z} w_{\bar{z} \underline{\underline{c}}}-1\right)\right] \\
& +\frac{\underline{a}+1}{\underline{c}+1}\left[(\underline{c}+1) w_{(\underline{a}+2)} w_{\bar{z} \underline{b}}{ }^{\underline{b}}+\partial_{z}\left(w_{(\underline{a}+2)} w_{\bar{z} \underline{b}} \underline{b-1}\right)\right] \delta^{\underline{a}+\underline{\underline{c}}} .
\end{aligned}
$$

After successive substitution the independent conformally covariant variables $w_{(\underline{a}+2)}$ together with their "duals,"

$$
v_{\bar{z}}-(\underline{a}+1)=w_{\bar{z} \underline{a}}-\underline{a}-1,
$$


will appear in differential expressions which are conformally covariant as a consequence of the inhomogeneous transformation law of the projective connection $\Lambda_{z z}$.

Let us illustrate this mechanism with the two simplest examples. For the value $\underline{c}=+1$ we obtain after straightforward calculation

$$
\partial_{\bar{z}} w_{(3)}-\mu_{\bar{z}}^{z} \partial_{z} w_{(3)}-3 w_{(3)} \partial_{\bar{z}} \mu_{\bar{z}}^{z}=\frac{1}{24} \Delta^{(5)} v_{\bar{z}}^{-2} \text {. }
$$

The covariant fifth order differential operator appearing here is given as

$$
\Delta^{(5)}=\partial^{5}+2 \partial^{3} \Lambda+10 \Lambda \partial^{3}+15 \partial \Lambda \partial^{2}+9 \partial^{2} \Lambda \partial+16 \Lambda \partial \Lambda+16 \Lambda \Lambda \partial .
$$

In the next complicated case, $\underline{c}=+2$, after slightly more tedious algebraic efforts one finds

$$
\partial_{\bar{z}} w_{(4)}-\mu_{\bar{z}}^{z} \partial_{z} w_{(4)}-4 w_{(4)} \partial_{\bar{z}} \mu_{\bar{z}}^{z}=\frac{1}{720} \Delta^{(7)} v_{\bar{z}}^{-3}+\frac{1}{15} Q^{(3)}\left(w_{(3)}, v_{\bar{z}}^{-2}\right) \text {. }
$$

Here, on the right hand side, two separately covariant differential expressions appear. The seventh order covariant differential operator comes out as

$$
\begin{aligned}
\Delta^{(7)}= & \partial^{7}+28 \Lambda \partial^{5}+70 \partial \Lambda \partial^{4}+84 \partial^{2} \Lambda \partial^{3}+56 \partial^{3} \Lambda \partial^{2}+20 \partial^{4} \Lambda \partial+3 \partial^{5} \Lambda+196 \Lambda \Lambda \partial^{3}+588 \Lambda \partial \Lambda \partial^{2} \\
& +352 \Lambda \partial^{2} \Lambda \partial+295 \partial \Lambda \partial \Lambda \partial+78 \Lambda \partial^{3} \Lambda+177 \partial \Lambda \partial^{2} \Lambda+288 \Lambda \Lambda \Lambda \partial+432 \Lambda \Lambda \partial \Lambda
\end{aligned}
$$

In addition, $Q^{(3)}\left(w_{(3)}, v_{\bar{z}}^{-2}\right)$ is a covariant expression containing $w_{(3)}$ and $v_{\bar{z}}^{-2}$ (and $\Lambda_{z z}$ of course) with derivatives $\partial_{z}$ up to third order

$$
\begin{aligned}
Q^{(3)}\left(w_{(3)}, v_{\bar{z}}^{-2}\right)= & 14 w_{(3)} \partial^{3} v_{\bar{z}}^{-2}+14 \partial w_{(3)} \partial^{2} v_{\bar{z}}^{-2}+6 \partial^{2} w_{(3)} \partial v_{\bar{z}}^{-2}+\partial^{3} w_{(3)} v_{\bar{z}}^{-2}+52 w_{(3)} \Lambda \partial v_{\bar{z}}^{-2} \\
& +18 \partial w_{(3)} \Lambda v_{\bar{z}}^{-2}+25 w_{(3)} \partial \Lambda v_{\bar{z}}^{-2}
\end{aligned}
$$

Clearly, at higher levels of $c$ the structure of the recursive solution which provides higher and higher covariant differential expressions requires more and more computational effort, calling for some algorithm which would allow to extract the results in closed form (possibly employing symplectic techniques as indicated in Ref. 26). This should also help to clarify the geometrical meaning of the zero curvature structures developed here, a question which undoubtedly deserves further study.

\section{SUPERSYMMETRY}

Turning to the supersymmetric case we consider the Lie algebra $\operatorname{OSp}(1 \mid 2)$

$$
\begin{gathered}
{\left[L_{k}, L_{l}\right]=(k-l) L_{k+l},} \\
{\left[L_{k}, G_{r}\right]=\left(\frac{k}{2}-r\right) G_{k+r},} \\
\left\{G_{r} G_{s}\right\}=2 L_{r+s},
\end{gathered}
$$

with indices $k, l \in\{-1,0,+1\}$ for the even generators and $r, s \in\left\{-\frac{1}{2},+\frac{1}{2}\right\}$ for the odd generators. In complete analogy with the bosonic case we define Lie algebra valued one-forms

$$
\Omega=M^{m} L_{m}+\Gamma^{r} G_{r}
$$

The corresponding field strengths 


$$
F=d \Omega-\Omega \Omega,
$$

are expanded in the same way, i.e.,

$$
F=F^{m} L_{m}+\Phi^{r} G_{r}
$$

Observe that in the supersymmetric case we are dealing with differential forms defined with respect to rigid superspace, i.e.,

$$
\Omega=e^{z} \Omega_{z}+e^{\bar{z}} \Omega_{\bar{z}}+e^{\theta} \Omega_{\theta}+e^{\vec{\theta}} \Omega_{\bar{\theta}},
$$

where the individual coefficients are superfields, i.e., functions which depend not only on coordinates $z, \bar{z}$ but on the anticommuting parameters $\theta, \bar{\theta}$ as well. Moreover, the frame of rigid superspace satisfies

$$
d e^{z}=-2 i e^{\theta} e^{\theta}, \quad d e^{\bar{z}}=+2 i e^{\bar{\theta}} e^{\bar{\theta}} .
$$

The field strength pertaining to the even and odd generators of the Lie algebra $\operatorname{OSp}(1 / 2)$ in our parametrization are given, respectively, as

$$
\begin{aligned}
& F^{-1}=d M^{-1}+M^{-1} M^{0}+\Gamma^{-1 / 2} \Gamma^{-1 / 2}, \\
& F^{0}=d M^{0}+2 M^{-1} M^{+1}+2 \Gamma^{-1 / 2} \Gamma^{+1 / 2}, \\
& F^{+1}=d M^{+1}-M^{+1} M^{0}+\Gamma^{+1 / 2} \Gamma^{+1 / 2},
\end{aligned}
$$

and

$$
\begin{aligned}
& \Phi^{-1 / 2}=d \Gamma^{-1 / 2}+M^{-1} \Gamma^{+1 / 2}-\frac{1}{2} M^{0} \Gamma^{-1 / 2} \\
& \Phi^{+1 / 2}=d \Gamma^{+1 / 2}+\frac{1}{2} M^{0} \Gamma^{+1 / 2}-M^{+1} \Gamma^{-1 / 2}
\end{aligned}
$$

In this general setting we now impose zero curvature conditions and establish, at the same time, the relation with the superspace Beltrami parametrization, using the conventions of Baulieu et al. ${ }^{16,17}$

We identify in particular

$$
\begin{aligned}
-\frac{i}{2} M^{-1} & \equiv M^{z}=e^{z}+e^{\bar{z}} M_{\bar{z}}^{z}+e^{\bar{\theta}} M_{\bar{\theta}}^{\bar{z}}, \\
-\frac{i}{2} \Gamma^{-1 / 2} & \equiv M^{\theta}=e^{\theta}+e^{\bar{z}} M_{\bar{z}}^{\theta}+e^{\bar{\theta}} M_{\bar{\theta}}^{\theta} .
\end{aligned}
$$

Moreover, adjusting consistently with the overall conformal weights, leads us to define

$$
M^{0} \equiv \chi, \quad \Gamma^{+1 / 2} \equiv \chi_{\theta} .
$$

The zero curvature conditions $F^{-1}=0$ and $\Gamma^{-1 / 2}=0$ then take the form

$$
\begin{gathered}
d M^{z}+M^{z} \chi+2 i M^{\theta} M^{\theta}=0 \\
d M^{\theta}+\frac{1}{2} M^{\theta} \chi+M^{z} \chi_{\theta}=0
\end{gathered}
$$


Again, the presence of the constant coefficients in $M^{z}$ and in $M^{\theta}$ allows to reduce the number of independent superfields. Straightforward manipulations yield (We use here the abbreviation $\partial_{z} M^{A}=e^{\bar{z}} \partial_{\bar{z}} M_{\bar{z}}^{A}+e^{\bar{\theta}} \partial_{\bar{z}} M_{\bar{\theta}}^{A}$ for $A$ equal $z$ or $\theta$.)

$$
\begin{gathered}
\chi=M^{z} \chi_{z}+\dot{d}_{z} M^{z}, \\
\chi_{\theta}=M^{z} \chi_{z \theta}+\frac{1}{2} M^{\theta} \chi_{z}+\partial_{z} M^{\theta} .
\end{gathered}
$$

Reinsertion of these relations in the zero curvature conditions discussed so far gives rise to equations where $\chi_{z}$ and $\chi_{z \theta}$ have dropped out and which then serve to express the superfields $M_{\bar{z}}^{z}, M_{\bar{z}}^{\theta}$, and $M_{\bar{\theta}}^{\theta}$ in terms of the independent superfield $M_{\bar{\theta}}^{z}$, as explained in great detail in Ref. 17.

At the next level we identify $2 i M^{+1} \equiv \lambda_{z}$ and write the zero curvature condition $F^{0}=0$ in the form

$$
d \chi+4 i M^{\theta} \chi_{\theta}+2 M^{z} \lambda_{z}=0
$$

This equation renders some of the coefficients of the superspace one-form $\lambda_{z}$ redundant, giving rise to

$$
2 \lambda_{z}=-d \chi_{z}+\left(\partial_{z} M^{z}\right) \chi_{z}+4 i M^{\theta} \chi_{z \theta}+\partial_{z} \partial_{z} M^{z}+M^{z}\left(2 \lambda_{z z}+\partial_{z} \chi_{z}\right)
$$

So far we have only encountered algebraic relations between superfields. This situation changes when we consider the last two zero curvature conditions $F^{+1}=0$ and $F^{+1 / 2}=0$ which, with the identifications introduced so far read

$$
\begin{aligned}
& d \lambda_{z}-\lambda_{z} \chi+2 i \chi_{\theta} \chi_{\theta}=0, \\
& d \chi_{\theta}-\frac{1}{2} \chi_{\theta} \chi+M^{\theta} \lambda_{z}=0 .
\end{aligned}
$$

These equations simply determine (somewhat loosely speaking) the $\theta$ and $\bar{\theta}$ components of the superfield $\chi_{z \theta}$. More explicitly one finds

$$
D_{\theta} \chi_{z \theta}=\frac{1}{2} \Lambda_{z z},
$$

i.e., the superfield

$$
\Lambda_{z z}=2 \lambda_{z z}+\partial_{z} \chi_{z}-\frac{1}{2} \chi_{z} \chi_{z}
$$

is identified in the $\theta$ component whereas at the $\bar{\theta}$ level we find the differential constraint

$$
D_{\overline{0} \chi} \chi_{z}=\frac{i}{4}\left(\partial_{z} \partial_{z} D_{\theta}+D_{\theta} \cdot \chi_{z \theta} D_{\theta}+4 i \partial_{z} \cdot \chi_{z \theta}+4 i \chi_{z \theta} \partial_{z}\right) M_{\vec{\theta}}^{z}
$$

This provides the supersymmetric generalization of the corresponding equation derived in the SL(2) case, involving the basic superfields $\boldsymbol{M}_{\vec{\theta}}^{z}$, which generalizes the Beltrami differential, and $\chi_{z \theta}$, which generalizes the projective connection. For a more detailed account of this construction and for the discussion of the structure of superconformal transformations we refer to Ref. 24.

In component field language we have $\mu_{\bar{z}}^{\bar{z}}$, the usual Beltrami differential and $\mu_{\bar{z}}^{\theta}$, its supersymmetry partner and, on the other hand, $\Lambda_{z z}$, the projective connection and $\chi_{z \theta}$, its supersymmetry partner (following widespread usage we employed here the same symbols for the superfields and their lowest components).

The corresponding component field equations are then 


$$
\partial_{\bar{z}} \Lambda_{z z}=\left(\partial_{z} \partial_{z} \partial_{z}+\partial_{z} \cdot \Lambda_{z z}+\Lambda_{z z} \partial_{z}\right) \mu^{\bar{z} z}-4 i\left(\partial_{z} \cdot \chi_{z \theta}+2 \chi_{z \theta} \partial_{z}\right) \mu_{\bar{z}}^{\theta}
$$

and

$$
\partial_{\bar{z}} \chi_{z \theta}=\left(\partial_{z} \partial_{z}+\frac{1}{2} \Lambda_{z z}\right) \mu_{\bar{z}}^{\theta}+\left(\partial_{\bar{z}} \cdot \chi_{z \theta}+\frac{1}{2} \chi_{z \theta} \partial_{z}\right) \mu_{\bar{z}}^{z}
$$

A crucial input in our construction of the supersymmetric case was the superfield version of the Beltrami parametrization, corresponding to the left-right decomposition of twodimensional superspace geometry. This means that two copies of the structures described here exist, related to each other by chiral conjugation. We expect that in the construction of analoguous systems with extended supersymmetry the corresponding Beltrami parametrizations will provide useful tools as well. Another interesting question is whether the supersymmetric procedure presented here can be extended to more general (and more complicated) super Lie algebras, in analogy with recent constructions of the super Toda theories. ${ }^{27}$

\section{CONCLUSIONS AND OUTLOOK}

We have proposed a constructive procedure to establish relations between simple Lie algebras and conformally covariant higher order differential operators via zero curvature conditions.

The basic ingredient is the decomposition of the basis of the Lie algebra in terms of representations with respect to the principal SL(2) subalgebra. The corresponding gauge structure exhibits then a number of intriguing properties. First of all, conformal covariance is established in assigning suitable properties under holomorphic coordinate transformations to the gauge potentials in the principal SL(2) subsector, where, in all cases, Beltrami differential and projective connection are made to come up. Then, at each spin $s(=\underline{a}+1$, where $\underline{a}>0$ in our notation) occuring in the Kostant decomposition of the Lie algebra we identify two conformally covariant fields $w_{(s+1)}$ and $v_{\bar{z}}^{-s}$ at highest and at lowest weight, respectively.

Conformally covariant constraints can be imposed in a suggestive way such that after taking into account the recursive structure of the zero curvature equations the independent covariant tensor variables are $\mu_{\bar{z}}^{z}, v_{\bar{z}}^{-s}$, and $w_{(s+1)}$, of conformal weights $-1, s$ and $s+1$, respectively.

As a final result, at each spin $s$ occuring, one is then left with an equation for $\partial_{z} w_{(s+1)}$ in terms of covariant differential expressions involving the basic covariant fields together with the projective connection $\Lambda_{z z}$ which ensures conformal covariance.

In particular, at each $s$, a covariant differential operator of order $2 s+1$ acting on $v_{\bar{z}}^{-s}$ emerges, mapping this tensor of conformal weight $-s$ into a conformal tensor of weight $s+1$. Other types of covariant differential expressions, involving the other covariant basic fields, and whose detailed form depends on the Lie algebra of interest, appear in a completely automatic way as well.

Although the constraints employed here are quite suggestive and have a lot of similarities with the constraint structure appearing in the WZNW construction of the Toda field theories, ${ }^{21,22,28}$ and their supersymmetric version, ${ }^{27}$ a better understanding should be achieved. In particular the remaining gauge invariances deserve further study. Recall that the constraints serve to eliminate the $d z$ components of the gauge potentials at each $s$ except for the highest weight one of conformal weight $s+1$.

As this kind of primary fields appears in the WZNW construction of the Toda theories as well, one might speculate whether the fields $v_{\bar{z}}^{-s}$, which appear here in a certain dual manner together with the $w_{(s+1)}$, may serve to formulate some kind of 'gauged' Toda system, thus providing a link with $W_{\mathrm{g}}$ gravity. ${ }^{29}$ 


\section{ACKNOWLEDGMENTS}

R. G. would like to thank F. Delduc, J. Madore, M. Saveliev, and in particular R. Stora for conversations on various aspects of the subject addressed here. We are grateful to L. Frappat, E. Ragoucy, and P. Sorba for advice concerning the SL(2) decomposition, for hints to the literature, and for introduction to the basics of WZNW constructions of the Toda theories.

'J. Weiss, J. Math. Phys. 27, 1293 (1986).

${ }^{2}$ W. Scherer, Lett. Math. Phys. 17, 45 (1989).

${ }^{3}$ P. Mathieu, in Integrable and Superintegrable Systems, edited by B. A. Kupershmidt (World Scientific, Singapore, 1990), p. 352-388.

${ }^{4} \mathrm{~L}$. Bonora and C. S. Xiong, Covariant $s l_{2}$ Decomposition of the $i_{n}^{*}$ Drinfeld-Sokolev Equations and $\boldsymbol{W}_{n}$ Algebras (SISSA/ISAS-187/90/EP, 1990).

${ }^{5}$ P. Di Francesco, C. Itzykson, and J.-B. Zuber, Commun. Math. Phys. 140, 543 (1991).

'J.-B. Zuber, KdV and W-flows, Saclay SPhT/91-052, 1991.

${ }^{7}$ V. G. Drinfel'd and V. V. Sokolov, J. Sov. Math. 30, 1975 (1984).

${ }^{8}$ J.-L. Gervais and A. Neveu, Nucl. Phys. B 209, 125 (1982).

9 J.-L. Gervais, Phys. Lett. B 160, 277 (1985).

${ }^{10}$ J.-L. Gervais, Phys. Lett. B 160, 279 (1985).

"B. A. Kupershmidt, Phys. Lett. A 109, 417 (1985).

${ }^{12}$ P. Mathieu, Phys. Lett. B 208, 101 (1988).

${ }^{13}$ A. Bilal, V. V. Fock, and I. I. Kogan, Nucl. Phys. B 359, 635 (1991).

${ }^{14}$ A. Das, W.-J. Huang, and S. Roy, Zero curvature condition and 2d gravity theories, Rochester UR1197, 1990.

${ }^{15}$ B. Kostant, Am. J. Math. 81, 973 (1959).

${ }^{16}$ L. Baulieu, M. Bellon, and R. Grimm, Nucl. Phys. B 321, 697 (1989).

${ }^{17}$ R. Grimm, Ann. Phys. 200, 49 (1990).

${ }^{18}$ R. Zucchini, A Polyakov Action on Riemann Surfaces (II), ENSLAPP-A-355/91, November 1991.

${ }^{19}$ D. Olive and N. Turok, Nucl. Phys. B 257, 277 (1985).

${ }^{20}$ B. R. Judd, Operator Technics in Atomic Spectroscopy (McGraw-Hill, New York, 1963).

${ }^{21}$ J. Balog, L. Fehér, L. O'Raifeartaigh, P. Forgács, and A. Wipf, Ann. Phys. 203, 76 (1990).

${ }^{22}$ L. Fehér, L. O'Raifeartaigh, P. Ruelle, I. Tsutsui, and A. Wipf, Ann. Phys. 213, 1 (1992).

${ }^{23}$ K. Schoutens, A. Sevrin, and P. van Nieuwenhuizen, Example of a quantum field theory based on a nonlinear Lie algebra, CERN-TH.6361/92, January 1992.

${ }^{24}$ G. Akemann, Diplomarbeit, Universität Hannover, May 1992.

${ }^{25} \mathrm{C}$. N. Pope, Lectures on $W$ algebras and $W$ geometry, CTP TAMU-103/91, December 1991.

${ }^{26}$ L. Baulieu, M. Bellon, and R. Grimm, Phys. Lett. B 260, 63 (1991).

${ }^{27}$ F. Delduc, E. Ragoucy, and P. Sorba, Commun. Math. Phys. 146, 403 (1991).

${ }^{28}$ A. N. Leznov and M. V. Saveliev, Commun. Math. Phys. 89, 59 (1983).

${ }^{29} \mathrm{R}$. Zucchini, Light cone $W_{n}$ geometry and its symmetries and projective field theory, Bologna DFUP.92, version 1 , May 1992. 